\title{
Le sentiment religieux en Nouvelle-France au XVII ${ }^{\mathbf{e}}$ siècle
}

\section{Guy-Marie Oury}

Volume 50, numéro 1, 1983

Bilan de l'histoire religieuse au Canada

Canadian Catholic History: A survey

URI : https://id.erudit.org/iderudit/1007046ar

DOI : https://doi.org/10.7202/1007046ar

Aller au sommaire du numéro

Éditeur(s)

Les Éditions Historia Ecclesiæ Catholicæ Canadensis Inc.

ISSN

0318-6172 (imprimé)

1927-7067 (numérique)

Découvrir la revue

Citer cet article

Oury, G.-M. (1983). Le sentiment religieux en Nouvelle-France au XVII ${ }^{\mathrm{e}}$ siècle. Sessions d'étude - Société canadienne d'histoire de l'Église catholique, 50(1),

255-279. https://doi.org/10.7202/1007046ar

Tous droits réservés @ Les Éditions Historia Ecclesiæ Catholicæ Canadensis Inc., 1983
Ce document est protégé par la loi sur le droit d'auteur. L'utilisation des services d'Érudit (y compris la reproduction) est assujettie à sa politique d'utilisation que vous pouvez consulter en ligne.

https://apropos.erudit.org/fr/usagers/politique-dutilisation/ 


\section{Le sentiment religieux en Nouvelle-France au XVII' ${ }^{\mathbf{e}}$ siècle}

Dans l'histoire des missions du XVIre siècle, trois ou quatre contrées semblent avoir été particulièrement fécondes en fruits de sainteté, sinon en résultats spectaculaires: le Japon, le Tonkin, le Canada, les Guaranis ${ }^{1}$. Mais ce n'est pas sous l'aspect missionnaire que nous avons à envisager ici le cas de la Nouvelle-France, puisqu'un atelier est consacré à l'Histoire des missions, mais sous l'angle de l'histoire de la spiritualité. Le titre choisi pour cette communication est commode en ce qu'il se réfere à l'ouvrage d'Henri Bremond, mais il prête aussi à confusion - outre son relent moderniste et anti-dogmatique - , car, dans le cas de la Nouvelle-France, son expression n'est pas seulement littéraire.

Le Canada a souvent fait peur aux Français de France; on menaçait les petits enfants de les y envoyer, afin d'obtenir de leur part un peu de sagesse; mais les âmes généreuses s'y sentaient attirées en grand nombre, comme en un lieu d'héroïsme et de sainteté, et son influence a été hors de proportion avec l'importance réelle de la mission, car, en réalité, les ouvriers apostoliques ont été limités en nombre; le plus petit diocèse de France, l'évêché le plus crotté, avait un clergé infiniment plus nombreux et un réseau de monastères autrement fourni.

Ce qui fait du Canada une réalité à part, une réalisation exceptionnelle de la Réforme catholique française, c'est la qualité spirituelle des hommes et des femmes qui se sont consacrés à la mission. L'historien de la spiritualité se doit d'en prendre acte, même s'il ne souscrit pas à la présentation des origines, telle qu'elle s'est élaborée au cours du $\mathrm{XIX}^{\mathrm{e}}$ siècle, sur le mode à la fois épique et mythique. On reste confondu devant la densité des personnalités exceptionnelles - dans l'ordre de la sainteté - qui ont oeuvré en Nouvelle-France au cours des premières générations. Le nombre des canonisés et béatifiés n'est pas,

\footnotetext{
${ }^{1}$ Voir S. Delacroix, Histoire des Missions catholiques, t. II. Les Missions modernes, Paris, 1957 (ouvrage collectif).
} 
à lui seul, un critère absolu: car la procédure favorise les martyrs et l'Église canadienne, aujourd'hui numériquement importante, a beaucoup travaillé pour faire avancer ses causes; mais le Canada des origines compte à lui seul un bon quart ou un tiers même des saints et bienheureux de l'Église de France au XVII ${ }^{\mathrm{e}}$ siècle. Il y a actuellement vingt saints français déjà canonisés pour ce siècle: huit ont vécu au Canada; il y a treize béatifiés dont deux ont vécu au Canada, auxquels il faut ajouter Kateri qui n'est pas française évidemment; parmi les vingt-deux causes de béatification actuellement en cours à Rome, deux appartiennent au Canada et deux autres lui sont étroitement liées (Marie de la Ferre et Jérôme Le Royer de la Dauversière). Bien entendu, la sainteté ne se limite pas à celle que l'Église a sanctionnée à la suite d'un procès ou que l'on voudrait faire reconnaître par elle; c'est cependant une indication positive qu'il serait vain de prétendre remplacer. L'historien, quelle que soit son appartenance, ne peut qu'en être impressionné; ce n'est pas là l'effet d'une simple coïncidence. Le nombre des causes introduites en Cour de Rome pour l'Espagne est sensiblement plus élevé que pour la France, et l'Italie est encore plus favorisée numériquement, avec une admirable constance jusqu'au milieu du XVIII ${ }^{\mathrm{e}}$ siècle: mais le rapport mission-métropole, pour utiliser des termes plus modernes, favorise certainement le Canada et en fait, sous cet angle particulier, une contrée exceptionnelle ${ }^{2}$.

La sainteté canadienne reflète évidemment celle du pays qui lui a envoyé ses missionnaires, et le cadre spirituel en est extraordinairement varié $^{3}$; le bloc formé par les martyrs jésuites présente sans doute une unité beaucoup plus cohérente à l'intérieur du groupe des saints de la Nouvelle-France, mais par ailleurs on retrouve au Canada toutes sortes d'influences qui se croisent et se mêlent, bien que tous, sans exception, aient bénéficié plus ou moins des mêmes méthodes de formation spirituelle, celle des Exercices de saint Ignace.

\footnotetext{
${ }^{2}$ Voir A. Rayez, "Marie de l'Incarnation et le climat spirituel de la NouvelleFrance", dans Revue d'Histoire de l'Amérique française, t. XVI, 1962, p. 3-34; on se reportera à la bibliographie de cette étude, très complète pour les travaux parus avant elle. On pourra aussi consulter l'article «France » dans le Dictionnaire de Spiritualité.

${ }^{3}$ Voir. R. Darricau, «La sainteté en France (1590-1715)», dans Histoire et Sainteté, $5^{e}$ rencontre d'Histoire religieuse, Angers-Fontevraud, 1981, Angers, 1982, p. 65-94; les autres communications présentent également un grand -intérêt dans la perspective qui nous occupe.
} 
On lit par exemple dans les Constitutions et Règlements des Premières Ursulines de Québec, de 1647:

Tant que faire ce poura, comme a esté dit ailleurs, les exercices spirituels se fairont une fois tous les ans par toutes les religieuses de la maison, en sorte qu'ils puissent estre faits devant la fin de l'octave de la Pentecoste, afin qu'en viron ce temps on puisse faire la solemnelle renovation des voeux. Ces exercices se prendront ordinairement de ceux qui ont esté composées par saint Ignace, fondateur de la Compagnie de Jésus, ou par quelqu'un de ses religieux, à qui il a pleu à Dieu en communiquer le ler esprit. ${ }^{4}$

Rien d'étonnant à cela, les Exercices qui ont été la matrice de la Compagnie de Jésus, ont été en même temps un instrument d'une grande efficacité pour la réforme de l'Église, et sont devenus en quelques décennies un bien commun à tous; on ne saurait compter le nombre des hommes et des femmes, des religieux et religieuses qui ont été remis par eux sur le chemin de leur vocation ou qui lui ont dû leur fidélité ${ }^{5}$.

Mais les Exercices de saint Ignace sont essentiellement un livre fonctionnel, décrivant et prescrivant une certaine manière de procéder, et proposant dans un ordre déterminé la progression d'une expérience spirituelle; c'est un ouvrage vivant, sans cesse soumis à des relectures et à des adaptations, afin de l'intégrer au contexte culturel d'une époque; le livre a été interprété différemment, sans pour autant être trahi. Rien de plus révélateur, à cet égard, qu'un des manuscrits conservé par les Ursulines de Québec, un recueil de Retraites, constitué probablement en 1650. On y trouve six éléments, tous inspirés et dérivés des Exercices de saint Ignace, et utilisés par les religieuses pour leurs temps de récollection: les huit jours avant la vêture, les exercices pour la profession ou pour sa rénovation, les retraites annuelles; le $6^{\mathrm{e}}$ élément propose un abrégé des Exercices de saint Ignace en trois journées et neuf méditations ${ }^{6}$.

On peut y voir, semble-t-il, une oeuvre collective dont une partie serait du Père Jérôme Lalemant, une autre, peut-être, de Mère Marie de

\footnotetext{
${ }^{4}$ Constitutions et Règlements des premières Ursulines de Québec, 1647, éd. G. Lapointe, Québec, 1974 (multigraphiée), p. 142.

${ }^{5}$ Saint Ignace de Loyola, Exercices spirituels, Texte définitif (1548), trad. et commentés par J.-Cl. Guy, Paris, 1982. Introduction.

${ }^{6}$ G.-M. Oury, "Le recueil des Retraites des Ursulines de Québec», dans Église et Théologie, t. 9, 1978, p. 271-89.
} 
Saint-Joseph, une autre enfin du Père Ragueneau; dont un recueil écrit au Canada, pour des religieuses canadiennes, dans la grande lignée des Exercices.

Les deux autres courants majeurs s'originent à saint François de Sales d'une part, à Bérulle, via Condren et $\mathbf{M}$. Olier de l'autre; il ne faut pas négliger non plus l'influence très réelle de Bernières, moins importante que les précédentes, mais profonde également, et celle de saint Pierre Fourier, limitée, elle, à Marguerite Bourgeoys et à son groupe de filles, où elle ne joue pas isolément, mais en conjonction avec celle de $\mathbf{M}$. Olier. On a donc ainsi cinq sources majeures qui rendent compte de la spiritualité vécue en Nouvelle-France au $\mathrm{XVII}^{\mathrm{e}}$ siècle, mais chacune d'elles ne se laisse pas isoler et a déjà conflué avec d'autres avant d'atteindre la mission du Canada. Le cas de Marie de l'Incarnation se situe à part, du fait de sa richesse et de son originalité propres, et nul ne saurait nier qu'elle ait exercé elle-même une influence profonde, non seulement sur les Ursulines de Québec, mais aussi sur les missionnaires eux-mêmes et sur les laïques qui l'ont beaucoup consultée.

Mise à part Marie de l'Incarnation, et à un moindre titre Catherine de Saint-Augustin, on ne peut parler à propos de la Nouvelle-France d'une floraison mystique, d'autant que les plus fondamentales des expériences de Marie de l'Incarnation se situent à une époque antérieure à son arrivée à Québec (1639); mais le Canada doit beaucoup aux mystiques, et nombre d'entre eux n'y sont jamais venus: Le Royer de la Dauversière, Olier, Marie Rousseau, Jean de Bernières, Gaston de Renty.

Certains historiens sont portés à négliger systématiquement leur importance; ce n'est pas de bonne méthode. Même si l'on se refuse à les considérer autrement que comme des croyants exaltés qui prennent leurs phantasmes pour des manifestations divines et agissent en conséquence, leur action doit être expliquée par leurs "inspirations", sous peine de ne pas être comprise; l'expérience mystique rentre ainsi par ce biais dans la matière historique, à titre de cause, et de cause d'une efficience tout à fait exceptionnelle en l'espèce.

En fait, pour nous, le mystique est un chrétien qui entend agir sous la motion de Dieu plutôt que de sa propre initiative et qui, dans ce but, se veut sans cesse attentif aux signes par lesquels se manifeste à lui cette volonté divine. Mais il n'est pas dépourvu de critique et nécessairement victime de ses illusions; il a appris à se défier de ses 
propres lumières, de son moi, de sa volonté, de ses désirs inavoués, de ses attraits, de son tempérament. Le mystique s'est soumis luimême à une ascèse exigeante qui l'a libéré de lui-même et de ses propres rêves, et il se fait toujours contrôler soigneusement par un «directeur" qui n'a aucun intérêt à se mettre à la remorque d'illusions, et est parfois tenté d'excès de sévérité et d'incrédulité systématique.

Une illumination intérieure, - qu'elle se présente sous forme de vision, de révélation, de songe même -, est souvent à l'origine de ce que le mystique réalise; mais elle est auparavant contrôlée par un tiers indépendant, soumise à un examen impitoyable destiné à éliminer tout ce qui serait illusion; les conseillers spirituels ne prennent au sérieux une illumination que si le bénéficiaire a fourni la preuve répétée de son authentique sainteté, par une vie pleinement conforme à l'idéal évangélique, et donc exempte de compromissions avec l'égoïsme et l'orgueil, sources habituelles des illusions ${ }^{\text {? }}$.

Sans doute, ce schéma est-il trop parfait pour avoir toujours été réalisé; un directeur prévenu en faveur de la mystique peut être un juge insuffisant, et l'on peut penser que le Père Ragueneau, par exemple, a péché parfois par excès de crédulité; mais le contrôle est habituellement très sérieux.

Le jugement d'une mystique, Marie de l'Incarnation, sur une autre mystique, Catherine de Saint-Augustin, montre clairement sur quels critères on opère le discernement, et avec quelle sévérité ont été passées au crible les «illuminations spirituelles";

De vous dire mon sentiment sur des matières si extraordinaires, je ne le puis, et je vous supplie de m'en dispenser, voyant que des personnes de science et de vertu y suspendent leur jugement et demeure dans le doute, n'osant se fier à des visions extraordinaires de cette qualité. Le Père Ragueneau y est savant et la tient pour bienheureuse, parce qu'elle a toujours été fidèle dans ses devoirs et qu'elle n'a jamais cédé au démon sur lequel elle a toujours été victorieuse. J'estime que cette fidélité dans ses obligations et dans ses combats la rendent grande dans le ciel et je m'y appuie plus volontiers que sur les visions que j'en entends dire. ${ }^{8}$

\footnotetext{
${ }^{7}$ Voir L. Cognet, Histoire de la Spiritualité chrétienne, sous la direction de L. Bouyer, t. III, Paris, 1965; L. Bouyer, Introduction à la vie spirituelle, Paris, 1960, p. 287-308; L. Gardet et $\mathrm{O}$. Lacombe, L'expérience du Soi, Étude de mystique comparée, Paris, 1981; voir surtout Introduction et p. 371-83.

${ }^{8}$ Marie de l'Incarnation, Correspondance, éd. G.-M. Oury, Solesmes, 1981, p. 887.
} 
Les sciences relevant de la psychologie ont fait des progrès depuis le $\mathrm{XVII}^{\mathrm{e}}$ siècle, mais celles que l'on possédait alors, jointes au discernement spirituel qui les transcende, ont été mises en oeuvre avec le plus grand scrupule dans la plupart des cas. On ne peut arguer, en tout cas, d'un manque d'esprit critique.

Le groupe des martyrs canadiens et plusieurs de leurs frères jésuites sont redevables: en partie de leur formation spirituelle au Père Louis Lallemant; le Père Paul Le Jeune a fait son troisième an sous sa direction; d'autres ont été ses novices, tels le Père Antoine Daniel et le Père Isaac Jogues; le Père Jean de Brébeuf semble l'avoir eu comme directeur spirituel. Les disciples les plus proches du Père Louis Lallemant ont été également en relation étroite avec les "Canadiens»; le Père Garnier a bien connu le Père Rigoleuc; le Père Chastelain a été frère de noviciat des Pères Huby et Maunoir, de sorte que les échanges ont été profonds et permanents ${ }^{9}$.

Bremond n'hésitait pas à faire entrer Louis Lallemant dans le groupe des Jésuites mystiques, au même titre le Père Surin, le Père Guilloré et le Père Saint-Jure, ce dernier ayant été en relation très intime avec le groupe des fondateurs de Montréal. Dans l'Histoire littéraire du sentiment religieux, en effet, il a cru discerner deux Écoles à l'intérieur de la Compagnie de Jésus en France, l'une officielle ou quasi-officielle qui donne la préférence à l'ascèse; l'autre, en marge, demandant à l'effort personnel un seul exercice, mais très exigeant, "la garde du coeur", dont le but est de supprimer les obstacles humains à l'action divine ${ }^{10}$; tandis que le Père Aloys Pottier, dans les trois volumes de son Essai de théologie mystique comparée sur le Père Louis Lallemant et ses disciples soutenait qu'il n'y avait entre les spirituels jésuites du XVII siècle que des "nuances insignifiantes" ${ }^{11}$. En fait, le Père Louis Lallemant n'est connu qu'à travers des notes d'auditeurs; son mysticisme est au confluent de nombreuses influences: celle du Père Balthazar Alvarez certainement, à l'intérieur de la tradition ignatienne; celle de sainte

\footnotetext{
${ }^{9}$ Voir. R. Rigault et G. Goyau, Martyrs de la Nouvelle-France, Extraits des Relations et Lettres des Missionnaires Jésuites, Paris, 1925; R. Rouquette, Textes des martyrs de la Nouvelle-France, Paris, 1947; F. Roustang, Jésuites de la Nouvelle-France, Textes choisis et présentés, Paris, 1960.

${ }^{10}$ Voir E. Goidrot, Henri Bremond, Histoire du Sentiment religieux, Genèse et stratégie d'une entreprise littéraire, Paris (1982); H. Bremond parle du Père Lallemant au tome III, Paris, 1920 , p. 64 ss.

${ }^{11}$ Voir A. Pottier, Le Père Louis Lallemant et les grands spirituels de son temps, Paris, 1927-1929.
} 
Thérèse, et peut-être celle de Benoît de Canfeld qui a marqué presque toute la génération de Bérulle; il a subi fortement l'influence des rhénoflamand, certainement à travers Harphius. En ce qui lui revient certainement, il a insisté sur l'abandon à la direction intérieure de l'EspritSaint, premier guide de l'âme, et sur l'importance de la contemplation. Il ne reprend aucun des thèmes centraux de Bérulle, contrairement à ce que pensait Bremond; mais il est exact que son mysticisme a inquiété le Père Général; le Père Mutius Vitelleschi lui reprochait dans une lettre du 9 avril 1629 d'être «tout mystique et de vouloir conduire tout le monde à une dévotion extraordinaire"; et l'on a mis un terme à son mandat d'instructeur du troisième an; mais son influence demeura grande dans la Compagnie ${ }^{12}$; le bérullisme que l'on relève dans la tradition issue de lui vient de ses disciples, tant les formules de Bérulle étaient devenues d'usage commun parmi les spirituels.

Le Jésuite de la mission canadienne le plus marquant par la mystique semble le Père Isaac Jogues; il y était prédisposé par sa grande sensibilité: ses grâces mystiques prennent la forme de rêves prémonitoires; il y a chez lui, avec l'héroïsme, quelque chose de profondément humain qui le rend très proche de tous: son affection, ses appréhensions; quand il va de la gloire de Dieu, il fait litière de ses craintes; il apaise l'affolement de son coeur et il fait montre d'une extraordinaire force d'âme: «Je serai heureux si Notre Seigneur voulait achever le sacrifice où il l'a commencé et que ce peu de sang que j'ai répandu en cette terre fût comme les arrhes de celui que je lui donnerai de toutes les veines de mon corps et de mon coeur» ${ }^{13}$.

Le Père de Brébeuf est aussi un mystique, mais plus serein, plus optimiste; son âme plane au-dessus des troubles, des causes d'inquiétude et d'anxiété, pourtant si nombreuses; il ignore l'impatience et la colère, comme s'il était déjà affranchi des passions communes, et la joie de la Résurrection se manifeste déjà dans les souffrances de la vie quotidienne; il vit dans la confiance et dans la joie; mais au-dessous, l'on devine les tentations de saint Antoine, qui l'ont plongé dans une humilité profonde; il est convaincu de sa nullité, malgré ses dons exceptionnels, il se croit inapte à la tâche à laquelle Dieu l'a appelé; toute son application et son désir sont de disparaître, et la nostalgie du paradis le

\footnotetext{
${ }^{12}$ Voir La Doctrine spirituelle du Père Louis Lallemant, éd. préparée par G.-M. Bertrand, Montréal-Paris, 1959; Louis Lallemant, Doctrine spirituelle, Introduction et notes de F. Courel, Paris, 1958.

${ }^{13}$ Lettre à un Jésuite, septembre 1646, dans Roustang, Jésuites, p. 271.
} 
travaille: "Je sens en moi une grande aversion de toutes les choses créées qu'il faudra quitter à la mort» ${ }^{14}$.

En mai 1630, il a promis de servir et a scellé dans son sang la cédule; puis il a fait le voeu de ne pas se dérober au martyre en 1637 et a prononcé le voeu du plus parfait en 1645 .

Un premier temps de son expérience mystique a été marqué par des visions démoniaques, incitant à l'infidélité et à l'apostasie; puis après 1640, celles-ci ont fait place à des visions de lumière êt de gloire à travers la souffrance: la Croix, la Vierge des Douleurs, la Crucifié lépreux lui donnant de partager son fardeau.

Saint Charles Garnier connaît sainte Lidwine dont il parle à son frère dans une lettre du 30 avril $1637^{15}$; il sait la dignité de l'état de victime pour les pécheurs ${ }^{16}$; il laisse cependant peu deviner à travers ses écrits l'aspect contemplatif de sa vie spirituelle et son chemin semble avoir été assez sombre. Il avait grande confiance en Marie de l'Incarnation. Saint-Gabriel Lallemant, lui, avait sollicité la grâce d'être envoyé en mission malgré son peu de santé, afin d'y souffrir, dit-il au Christ, «pour le salut des âmes que vous estimez vôtres, qui ont coûté votre sang, que vous avez aimées jusqu'à la mort...".

Le désir si vif de la mission s'est évanoui en arrivant en NouvelleFrance pour le Père Noël Chabanel; tout lui est devenu extrêmement pénible et il a eu la tentation continuelle de demander son rappel en France jusqu'à ce qu'il ait prononcé en 1647 le voeu de stabilité dans les missions.

Mais il n'y a pas que les martyrs chez les Jésuites, et tous en avaient plus ou moins le désir. Le Père Paul Le Jeune s'est laissé désigner pour la Nouvelle-France, sans rien demander, mais il était prêt à tout ce que Dieu voulait de lui: "Ecce me, me voilà tout entier entre les mains (de mon provincial) et pour le Canada, et pour la France, et pour tout le monde ad majorem Dei gloriam. Je me vois si faible à tout, et Dieu si puissant pour tout, qu'il me semble qu'il n'y a plus rien à désirer si à refuir» ${ }^{17}$, libre et joyeux, disponible pour le

\footnotetext{
14 «Manuscrits autobiographiques *, dans Roustang, Jésuites, p. 128.

${ }^{15}$ Roustang, Jésuites, p. 294.

${ }^{16}$ Lettre du 31 mars 1636, ibid., p. 289.

${ }^{17}$ Roustang, Jésuites, p. 42.
} 
service de Dieu, comme Dieu voudra. En fin de la Relation de 1635, il a laissé par écrit «Divers sentimens et Avis des Pères qui sont en la nouvelle France» ${ }^{18}$. Marie de l'Incarnation semble avoir médité ce texte dans lequel on trouve plusieurs des expressions dont elle usera; l'un et l'autre se retrouvaient dans l'importance donné au sang du Christ.

L'amour de la Nouvelle-France a été l'inspiratrice de la vie du Père Massé, premier ouvrier de la mission en Acadie, qui, selon Marie de l'Incarnation, a été «tout sainte»:

Si Jacob a servi quatorze ans pour Rachel, à combien plus forte raison dois-je servir mon cher Maître deux fois sept ans pour la Nouvelle France, mon cher Canada, embelli d'une grande variété de croix très aimables et très adorables? Un si grand bien, un si grand emploi, une vocation si sublime, en un mot le Canada et ses délices qui sont la croix, ne se peuvent obtenir que par des dispositions conformes à la croix. ${ }^{19}$

Les témoignages d'une vie mystique authentique abondent parmi les Jésuites de la mission. Le Père Chastellain, confident de Marie de l'Incarnation, a composé en Huronie durant les longs mois d'hivernage une série de Méditations sur la vie de Notre Seigneur: l'Affectus amantis Jesum Christum, qui dénotent un sens aigu des perspectives apostoliques de toute vie chrétienne et de l'appel universel au salut; et l'on peut en rapprocher telle ou telle formule des écrits de Marie de l'Incarnation: "Que ton Esprit devienne double en moi, humain et divin, intérieur et extérieur» ${ }^{20}$. Il sait ce qu'est, pratiquement, la contemplation dans l'action. Et sa dévotion à l'Eucharistie a pris des formes touchantes et enfantines: si le sommeil le terrassait dans la journée en Huronie, il allait faire un petit somme devant le Saint-Sacrement, "non sans un grand sentiment de piété... et tandis qu'il dormait, (il gouûtait) parfois des délices célestes".

Le Père Chaumonot avait appris l'humilité d'une enfance bohême et aventureuse, et celle-ci l'avait conduit à une voie d'enfance spirituelle, remplie de foi, de confiance en Dieu, de simplicité de coeur.

Marie de l'Incarnation a beaucoup apprécié la direction du Père Jérôme Lalemant dont la langue fourmille d'archaïsmes savoureux; il avait le sens du concret, de l'image parlante. Si l'attribution des trois

\footnotetext{
${ }^{18}$ Ibid., p. 72-82.

- ${ }^{19}$ Notes spirituelles, dans Roustang, Jésuites, p. 91.

${ }^{20}$ Roustang, Jésuites, p. 158.
} 
premières Retraites du recueil des Ursulines doit être retenue, il semble avoir aimé particulièrement les thèmes de la mort au vieil homme, de l'Imitation du Christ dans tous les états de sa vie, du mérite et de l'efficience de son Sang, de l'Eucharistie; la première Retraite se développe autour d'une théologie de l'image de Dieu, défigurée par le péché, rétablie par la conformité avec Celui qui est l'Image même, le Verbe de Dieu incarné; il faut, après avoir dépouillé tout ce qui relève du vieil homme se revêtir de l'humilité de coeur de Jésus-Christ, de sa pauvreté, de sa charité, de sa miséricorde, de sa douceur et de sa mansuétude, de sa mortification, de sa ferveur et dévotion, de sa prudence et de sa discrétion. La profession est une mort, par la triple immolation des voeux, d'où Dieu fait jaillir la grâce, de la double source du Verbe Incarné et l'Esprit-Saint; l'âme doit désormais se conformer aux États du Verbe, spécialement à sa vie obscure et cachée au ventre de sa Mère, et à sa vie conversante. On reconnaît là des thèmes à saveur bérullienne; mais la perspective de l'auteur reste ascétique et volontariste $^{21}$.

Le Père Paul Ragueneau est l'un des Jésuites du Canada que l'on connaît le mieux par ses écrits, car il s'est fait le collecteur de textes spirituels et sa correspondance est riche. Il eut le Père Louis Lallemant comme préfet des études en 1633, puis comme recteur en 1634-1635. Si la troisième série des Retraites des Ursulines lui revient, il faut y voir l'expression de sa fidélité extrême et de son recours direct à la tradition ignatienne des Exercices; les auteurs qu'il recommande sont Rodriguez, Stella, mais aussi le Père Saint-Jure, grand ami et directeur du baron de Renty et de Jeanne Mance, un Jésuite mystique qui n'a cessé de prêcher l'abandon à la divine Providence et à la conduite de l'Esprit de Dieu. De même le Père Ragueneau se montre-t-il très attentif à cette conduite, recommandant bien de n'en pas entraver l'action par des initiatives personnelles. La source de la participation aux souffrances rédemptrices du Christ a son lieu privilégié dans la célébration de la Messe. S'il fallait attribuer cette partie au Père Le Mercier, on constaterait une fois de plus les convergences. Les Jésuites du Canada ont beaucoup regardé du côté de leurs frères de France qui proposaient un enseignement résolument mystique, tout en demeurant essentiellement fidèle à la tradition ignatienne. Le Père Ragueneau met au service de celle-ci son bon sens, son expérience, sa connaissance des âmes, son souci de s'adapter à la disposition et à la situation particulière de chacun. Avec force, mais dans la paix et la tranquillité, il propose toujours

${ }^{21}$ G.-M. Oury, «Le recueil des Retraites », p. 276 s. 
aux âmes le motif déterminant de l'amour de Dieu; son style même a quelque chose qui rappelle l'Imitation de Jésus-Christ ${ }^{22}$.

Marie de l'Incarnation a laissé sur les Jésuites de la Mission du Canada des jugements qui sont une reconnaissance très explicite de leur sainteté; on pourrait aisément multiplier les citations et constituer un florilège. Elle savait ce qu'elle disait. Il n'y a pas à se méfier ici du parti-pris des gens de dévotion de ne dire que le bien et de relever le positif partout où ils le rencontrent, en passant sous silence des lacunes trop évidentes. Marie de l'Incarnation est un juge suffisamment averti pour que l'on ne se méprenne pas sur sa pensée, et tel jugement parfois sévère, mais toujours nuancé et pesé, ne permet pas de recuser ceux qui sont tout de louange; ces derniers abondent sous sa plume, dès qu'il est question des missionnaires: «Tous les Pères de la Compagnie se rendent admirables par leurs actions héroïques : ils ne craignent ni vie ni mort, se jetant par un saint aveuglement dans la barbarie la plus féroce" ${ }^{23}$. Et elle se fâche quand on se permet de critiquer leur emprise sur la colonie.

La physionomie de Marie de l'Incarnation domine de très haut l'univers spirituel canadien du milieu du XVII ${ }^{\mathrm{e}}$ siècle, comme elle occupe une place très éminente parmi les mystiques français de son siècle ${ }^{24}$. Depuis que s'est dessinée sa vocation canadienne en 1634-1635, elle est sous la direction de Jésuites et entretient avec eux d'étroits rapports; il y a eu quelques ombres, mais sa dette est grande à l'égard du Père de la Haye, du Père Dinet, du Père Saint-Jure, du Père Jérôme Lalemant surtout, du Père Le Mercier et du Père Ragueneau; elle connaît aussi et aide les jeunes Jésuites de la Mission, exerçant à leur égard une sorte de maternité spirituelle, dès avant son arrivée en NouvelleFrance en 1639.

Mais sa formation spirituelle ne doit rien aux Pères de la Compagnie; c'est dans un autre univers qu'elle a évolué au temps où s'est formée sa vie intérieure et où s'est nouée son expérience spirituelle. Durant le temps de ses grandes expériences fondamentales, elle a été guidée par des moines, les Cisterciens de la petite Congrégation réformée des Feuillants. Par eux, elle a été mise en contact avec les écrits de saint François de Sales, de sainte Thérèse d'Avila, de saint Jean de la Croix, du Pseudo-Denys.

\footnotetext{
${ }^{22}$ Voir J. Saint-Antoine, Paul Ragueneau et ses lettres spirituelles, Montréal, 1974.

${ }^{23}$ Marie de l'Incarnation, Correspondance, p. 124.

${ }^{24}$ Voir l'article «Marie de l'Incarnation» du Dictionnaire de spiritualité et la bibliographie.
} 
Elle éprouve beaucoup d'attrait pour les thèmes bérulliens, mais elle est trop jeune pour avoir bénéficié de l'amitié et de l'influence de sa grande compatriote, la Carmélite Madeleine de Saint-Joseph, disciple préférée de Bérulle; ce qu'elle connaît du bérullisme lui est venu sans qu'elle l'ait cherché, à travers les prédications. Il y avait en effet à Tours une maison de l'Oratoire, fondée au temps où elle était jeune femme, et le Père Métezeau y a résidé plusieurs années; les deux frères Gault étaient originaires de Tours; on sait la place qu'ils ont tenu dans l'Oratoire. Le Supérieur des Ursulines de Tours, Dominique Sain ${ }^{25}$, était également très pénétré de la doctrine de Bérulle: sens de l'adoration des grandeurs de Dieu, christocentrisme, culte du mystère de l'Incarnation, attention portée à la vie cachée et à l'intérieur de Jésus; mais son ascèse, celle qu'il proposait aux âmes, avait un caractère moins passif et, pour ainsi dire, moins mystique que celle de Bérulle; il recommandait la docilité à la grâce, l'attention aux lumières et aux inspirations de Dieu, mais non de laisser agir le Christ seul dans l'âme, en maintenant celle-ci dans l'état de servitude. Plusieurs de ses formules présentent des analogies avec celles de Pascal. Un des signes de bérullisme chez Marie de l'Incarnation est son voeu de servitude à Marie, dont elle a porté le signe sous la forme de la petite chaîne du saint esclavage qu'elle avait au cou et qu'elle a léguée à son fils en mourant.

Marie de l'Incarnation n'a rien d'une théoricienne de la spiritualité et des états mystiques; elle a décrit simplement son expérience pour ceux qui étaient chargés de la diriger, en 1633 pour le Père de la Haye, en 1653-1654 pour le Père Jérôme Lalemant sur les instances réitérées de son fils; de ce fait, elle a dû réfléchir pour mieux rendre compte de ce qu'elle avait vécu et de ce qu'elle vivait, mais toujours dans un but immédiatement pratique. Elle a été plusieurs fois déconcertée par toutes les questions que pouvait se poser son fils quand il recevait communication de ce qu'elle avait expérimenté; elle se contentait pour elle-même de décrire, sans souci de mettre en forme son expérience à l'intention des autres; son expérience-type, un modèle auquel on devrait nécessairement se référer. Cela ne l'empêchait pas, à l'occasion, de donner des conseils extrêmement judicieux, ou même de tenter, comme dans la finale de la Relation de 1654, de rechercher l'unité profonde de son aventure intérieure, en la ramenant à une idée fondamentale, la pauvreté spirituelle.

\footnotetext{
${ }^{25}$ Voir G.-M. Oury, «Les Retraites spirituelles de Dominique Sain, théologal de Tours », dans Revue d'Ascétique et de Mystique, t. XLV, 1969, p. 65-78.
} 
Quoi qu'il en soit, elle est très différente des maîtres qui enseignent, des théoriciens qui bâtissent. Si elle est maître spirituel, c'est à son insu. Elle ne crée pas une école et ne se conforme pas à une école ${ }^{26}$.

De l'école rhéno-flamande, elle a hérité cependant d'un sens extrêmement aigu de la transcendance divine, que son expérience personnelle a encore rendu plus profond; elle emploie fréquemment, comme les maîtres de l'école, les formes superlatives, comme suressentiel, suréminent. L'union à Dieu selon l'essence, est en fait pour eux comme pour elle une union selon la volonté, car «l'essence» d'un être conscient se résume, se ramasse et se concrétise en quelque sorte dans sa volonté; l'union s'accomplira quand la volonté de l'homme qui appartient au monde du néant et des apparences, ira se perdre dans volonté divine, quand elle s'absorbera en elle. Les rhéno-flamands se demandaient si l'humanité du Christ était comprise parmi les éléments créés que l'âme devait dépasser pour se perdre en Dieu; ils ont répondu généralement par l'affirmative, et il fut un temps où Marie de l'Incarnation ellemême ne se sentait plus libre de s'arrêter à l'humanité du Christ. Mais les partisans de ce dépassement étaient cependant unanimes à considérer que l'humanité du Verbe Incarné était le seul chemin qui conduise à la divinité; Bérulle a insisté dans ce sens, et Marie de l'Incarnation a trouvé sur ce point un merveilleux équilibre dans son culte et sa contemplation de la double beauté, divine et humaine du Verbe, devenu le plus beau d'entre les enfants des hommes.

Avec les maîtres rhéno-flamands, Marie a senti que l'union à Dieu, parce qu'immédiate, est une union sans entre-deux, sans aucun intermédiaire, même d'ordre intellectuel; elle a expérimenté la nécessité pour elle de dépasser les pensées, les réflexions, les images, les connaissances, qui risquaient d'entraver son progrès spirituel. Elle n'est pas anti-intellectuelle, mais, en fait, son expérience de Dieu se situe dans un au-delà. Au Père Poncet, elle confie en 1670: «Je me sens dans une pauvreté qui m'anéantit sous son poids aux pieds de sa divine Majesté. Avec tout cela Dieu fait compâtir avec cet état celui d'union qui me tient liée à sa divine Majesté, il y a plusieurs années, sans en sortir un seul moment... Dans cet état, l'âme possède tous les mystères, mais par une seule et simple vue, car d'y faire des réflexions, cela lui est impossible: la pensée des Anges et des Saints ne peut être que

\footnotetext{
${ }^{26} \mathrm{~L}$. Cognet, Les origines de la spiritualité française, Paris,s 1949; it., De la dévotion moderne à la spiritualité française, Paris, 1958; et le t. III de l'Histoire de la Spiritualité chrétienne (Sous la direction de L. Bouyer).
} 
passagère, car, en un moment et sans y penser, elle oublie tout pour demeurer dans ce fond où elle est perdue sans aucune opération des sens intérieurs" ${ }^{27}$.

Et elle précise la même année, à l'intention de son fils :

Je me vois perdue par état dans sa divine Majesté, qui, depuis plusieurs années, me tient avec elle dans un commerce, dans une liaison, dans une union et dans une privauté que je ne puis expliquer. C'est une espèce de pauvreté d'esprii qui ne me permet pas même de m'entretenir avec les Anges, ni des délices des Bienheureux, ni des mystères de la foi. Je veux quelquefois me distraire moi-même de mon fond pour m'y arrêter et m'égayer dans leur beauté comme dans des choses que j'aime beaucoup; mais aussitôt je les oublie, et l'esprit qui me conduit me remet plus intimement dans mon fond où je me perds dans Celui qui me plaît plus que toutes choses. J'y vois ses amabilités, sa Majesté, ses grandeurs, ses pouvoirs, sans néanmoins aucun acte de raisonnement et de recherche, mais en un moment qui dure toujours... ${ }^{28}$

La contemplation est donc absolument libérée des images et n'est plus en état d'utiliser le raisonnement discursif; les rhéno-flamands parlaient de nudité d'esprit, Marie de l'Incarnation préfère le terme de pauvreté en son sens le plus plein; elle s'est longuement expliqué làdessus dans le chapitre conclusif de la Relation de 1654 où elle résume son expérience antérieure en terme d'entrée dans l'état de pauvreté, de dépouillement, de nudité, de désappropriation, d'oubli d'elle-même. Elle est bien convaincue de son néant, mais maintient fermement, contre les excès du néantisme, que ce néant est un néant propre au Tout, proportionné à lui, destiné à lui, et que Dieu a la plus grande considération pour ce néant. Le caractère d'union mystique dans une fête d'épousailles qui a marqué de manière indélébile son expérience, et qui lui a donné conscience de son état d'épouse du Verbe incarné, l'a préservée également des excès de certaines formules où la créature semble annihilée en Dieu.

Mais, comme les rhéno-flamands, sa volonté humaine est et se veut pleinement absorbée dans la volonté divine, et sa contemplation est plus un état qu'un acte transitoire; elle a expérimenté qu'elle vivait en Dieu et que Dieu vivait en elle.

${ }^{27}$ Correspondance, p. 888.

${ }^{28}$ Correspondance, p. 897. 
Les points de rencontre avec l'héritage de la spiritualité nordique sont donc nombreux chez Marie de l'Incarnation, beaucoup plus nombreux qu'avec les mystiques espagnols, ou même avec Bérulle.

Du fait de sa description de la contemplation comme état plutôt que comme acte, elle a pu servir à Mme Guyon d'argument, en faveur d'un certain type de relation à Dieu, comme d'ailleurs la même se réclamait de sainte Jeanne de Chantal. Mme Guyon avait en outre consulté le fils de l'Ursuline, Dom Claude Martin, avant d'entreprendre une mission qui, comme pour Marie de l'Incarnation, devait l'éloigner de ses enfants: la fondation à Gex d'une maison de Nouvelles Catholiques. Les Justifications de Madame Guyon, composées en 1695, à l'occasion des conférences d'Issy, utilisent, avec saint Jean de la Croix, Olier, Benoît de Canfeld, Jean de Saint-Samson, Louis Épiphane, abbé d'Etival, et Marie de l'Incarnation; elle les envoya à Bossuet le 3 octobre. Fénelon invoquait Marie de l'Incarnation à l'appui de ses thèses, et Bossuet, pour ne pas demeurer en reste, l'a revendiquée de son côté ${ }^{29}$.

Mais elle ne se laisse pas facilement confisquer; son expérience lui est particulière; par plus d'un trait, elle pourrait être comparée à celle de Claudine Moine, qui rédigea précisément ses relations spirituelles à Paris dans les mêmes années, de 1652 à $1655^{30}$. Ce qui frappe chez elle, c'est l'équilibre, l'unité profonde réalisée au sein d'une existence apparemment tiraillée entre deux pôles opposés: la contemplation mystique, de l'ordre le plus élevé, et une action extérieure multiforme et exigeante qui semble ne lui laisser que des loisirs infimes, et ne pas permettre une liberté d'esprit suffisante.

Elle a déclaré elle-même dans une lettre de 1665 que l'intégration de l'action et de la contemplation progressait avec les différentes étapes de la vie intérieure; déjà dans l'état très élevé qu'elle nomme l'oraison d'union: «Si la personne a de grandes occupations, dit-elle, elle y travaille sans cesser de pâtir ce que Dieu fait en elle: cela même la soulage, parce que les sens étant occupés et divertis, l'âme en est plus libre; d'autres fois, les affaires temporelles et la vie même lui sont extrêmement pénibles à cause du commerce qu'elles l'obligent d'avoir avec les créatures»; mais dans l'état du mariage spirituel et mystique,

\footnotetext{
${ }^{29}$ Voir L. Cognet, Le Crépuscule des mystiques, le Conflit Fénelon-Bossuet, Tournai, 1958, p. 66, 68, 248 et 315.

${ }^{30}$ Voir La couturière mystique de Paris, Claudine Moine, Relations spirituelles, présentation par J. Guennou, Paris, 1981.
} 
l'intégration est parfaite: "Les sens y sont tellement libres que l'âme qui y est parvenue peut agir sans distraction dans les emplois où sa condition l'engage» ${ }^{31}$.

Chez elle, cette synthèse s'est réalisée bien avant son arrivée au Canada, et son nouveau directeur spirituel de 1645 , le Père Jérôme Lalemant ne pouvait que la constater avec joie: «(Il)... me disait que je ne devais jamais refuser l'emploi dans les affaires temporelles, vu qu'elles ne me distrayaient point du grand commerce dont il plaisait à ia divine Majestế $m^{\prime}$ honorer avec elle" ${ }^{3 \hat{z}}$.

Marie de l'Incarnation au Canada a été contemplative dans l'action; elle apportait dans son apostolat un don d'oraison extraordinaire qui lui permettait même de faire de l'étude des langues indiennes, pourtant si ardues - elle le reconnaît elle-même - une oraison. Cette unité de l'action et de la contemplation en laquelle elle a réalisé en plénitude sa vocation missionnaire, produisait en elle, disait-elle, une paix suréminente qui était la marque certaine de la profondeur de cette unité.

"Monsieur de Genève dit qu'il y a des oiseaux qui, en volant, prennent leur réfection. J'en suis de même en matière de la vie de l'esprit, car dans les tracas où je suis attachée par nécessité, je prends la nourriture solide et continuelle que je viens de dire" ${ }^{33}$. Par son exemple, Marie de l'Incarnation a prouvé que la vie apostolique engagée dans les tâches les plus accaparantes et les plus distrayantes peut prendre appui sur la vie contemplative et alimenter la contemplation elle-même; le coeur est fixé en Dieu, il adhère à lui et le contemple par la médiation même de l'action apostolique; le seul mélange qui fasse obstacle à la vraie contemplation est le mélange de soi-même, l'impureté qui fait que l'être en son intime n'appartient pas réellement et totalement à Dieu :

Notre union n'est jamais plus éminente que dans les travaux soufferts à l'imitation et pour l'amour de Jésus-Christ qui était dans le temps de ses souffrances et surtout au point de sa mort dans le plus haut degré d'union et d'amour pour les hommes avec Dieu son Père.... ${ }^{34}$

La loi d'unité régit la vie spirituelle de Marie de l'Incarnation, et pas à ce plan seulement; son entrée dans la vie mystique à l'âge de

\footnotetext{
${ }^{31}$ Marie de l'Incarnation, Correspondance, p. 748.

32 Ibid., p. 317.

${ }^{33}$ Ibid., p. 641.

${ }^{34}$ Ibid., p. 397.
} 
vingt ans, le 24 mars de l'année 1620 , a été préparée par une grâce de même ordre qu'elle a eue au moment de son entrée dans l'âge de raison. En 1620, elle a reçu comme une investiture: elle a été plongée dans le Sang du Christ et a découvert ce que représentait le fait de la Rédemption pour elle comme pour chacune des âmes; elle aura toujours devant les yeux le prix de la Rédemption et la hantise que ce Sang ait été répandu en vain. Toutes les âmes acquises par le Sang du Christ doivent devenir effectivement sa possession, son royaume; elle a conscience du caractère tragiquement sérieux de l'Amour divin, de ses droits infinis et méconnus. Dieu est Dieu et l'on ne se moque pas de lui; il lui faut toute la place en elle-même, sans partage, et toutes les âmes sont appelées de même à la sainteté. Et chacune des grâces de son itinéraire mystique: les illuminations trinitaires, l'union matrimoniale avec le Verbe ont une orientation apostolique qui se dessinera dans toute sa netteté quand elle aura fait choix, sous la poussée de l'Esprit, de la vie religieuse chez les Ursulines, à un âge où son jeune fils aurait eu encore besoin d'elle pour quelques années encore.

Sans verser dans le paradoxe, on peut affirmer à la fois le caractère classique de l'expérience spirituelle de Marie de l'Incarnation et son originalité. Elle présente des affinités avec Thérèse d'Avila, Jean de la Croix, Bérulle, Bernières, et plus lointainement avec Catherine de Sienne; mais même là où les affinités sont le plus sensibles, il n'y a pas de dépendance, et c'est toujours "après coup", après avoir fait ses propres expériences, qu'elle aborde les auteurs qui auraient pu la guider et où elle a la joie de se reconnaître partiellement. Antérieurement à son entrée dans la vie mystique, elle a eu la dévotion, répandue en Touraine, aux Cinq plaies du Christ, héritage médiéval, consacrée sur place par saint François de Paule, venu à Tours à la demande de Louis XI. Sa dévotion au Sang du Christ, puis au Coeur du Christ, la première datant de la grâce du 1620 , la seconde naissant vers 1625 , se résument dans son culte pour la Personne du Sacré Verbe Incarné dans sa double beauté divine et humaine.

Les grâces mystiques lui ont été accordées sous une forme en harmonie avec la structure de son esprit; l'élément intellectuel prédomine sur tout autre; la sensibilité n'a presque pas de place et les images n'apparaissent que rarement, le plus souvent sous la forme de l'allégorie. Elle pratique l'introspection; ses analyses sont d'une lucidité et d'une précision remarquables; mais elle n'aime pas les complications inutiles et coupe court aux réflexions vaines et superflues. La simplicité qu'elle prêche est chez elle qualité de nature avant de devenir vertu. 
Son itinéraire mystique est absolument normal, normatif pourrait-on dire; en dépit de son caractère exceptionnel, il offre le type parfait de la voie par laquelle l'Esprit Saint fait monter progressivement les âmes qui s'adonnent à sa conduite. Au plan de la conscience et de l'expérience, ce qu'elle vit est tout simplement l'essentiel des réalités de la vie chrétienne: la grâce de filiation donnée au baptême, l'union mystique, comparable au mariage que toute âme contracte avec Dieu quand elle lui est unie par la grâce, ou qu'elle s'engage plus totalement à son service par la consécration religieuse, l'habitation de la Trinité dans l'âme du fait de la vie théologale qui lui est donnée dans les sacrements, l'union au Verbe Incarné dont l'Eucharistie est le moyen privilégié. Tout chez elle est à la fois exceptionnel et pourtant en parfaite cohérence avec tout ce que le Nouveau Testament enseigne de la vie en Dieu, dans le Christ et dans l'Esprit qui est le partage de toute âme chrétienne.

Lorsque l'on quitte l'univers spirituel de Marie de l'Incarnation pour celui de Catherine de Saint-Augustin, l'impression est une douloureuse plongée dans les ténèbres ${ }^{35}$. Les "diableries" sont pratiquement absentes de l'expérience intérieure de l'Ursuline, sauf de très loin en très loin. Ici, ils sont omniprésents. Sur un mode différent et dans des circonstances autres, on retrouve en Catherine de Saint-Augustin quelque chose de l'étrange sainte de Coutances, Marie des Vallées, vénérée par le Père Louis d'Argentan, par Bernières, par saint Jean Eudes surtout; une malédiction semble peser sur elle; elle est comme une sorte de bouc émissaire pour les péchés de sa génération qu'elle porte en elle sous forme de tentations et d'obsessions ${ }^{36}$.

Mais alors que Marie des Vallées vit comme une pauvresse, parcourant les routes, vêtue de haillons, se comportant extérieurement, à certaines périodes de sa vie, comme une possédée, et connaissant même les prisons de l'officialité, il ne paraît rien à l'extérieur de l'aventure que mène Catherine de Saint-Augustin; au milieu de ses soeurs, elle vit très simplement, remplissant la charge de dépositaire, de première hospitalière, de maîtresse des novices, aimée des malades pour sa gentillesse et son sourire; on songeait à l'élire pour supérieure, quand

\footnotetext{
${ }^{35}$ Voir. P. Ragueneau, La Vie de la Mère Catherine de Saint-Augustin, Paris, 1671; G. Boucher, Dieu et Satan, Paris, 1979; A. Merlaud, L'épopée fantastique d'une jeune Normande, Catherine de Longpré, Paris, 1981.

${ }^{36}$ Voir. E. Dermenghen, La vie admirable et les révélations de Marie des Vallées, Paris, 1926.
} 
elle mourut le 8 mai 1668 à 36 ans d'un crachement de sang. Le Père Ragueneau publia sa Vie en 1671 à partir de relations autobiographiques; dès après sa mort on avait commencé à parler à son sujet de faits extraordinaires. Joyeuse, sereine, paisible pour son entourage, elle avait été en fait obsédée du démon, tourmentée de mille manières, sentant peser sur elle tous les péchés du Canada et les voyant en quelque sorte installés en elle; elle avait vécu en compagnie des anges noirs, s'offrant généreusement à Dieu pour tous les genres de supplice et de tentation, pourvu que le péché en tant qu'acte volontaire ne trouve pas de connivence en elle. Les tentations contre la chasteté ont été particulièrement rudes chez elle. Elle s'est donnée comme victime pour protéger le Canada et obtenir pour lui miséricorde.

Quant à la Mère de Saint Augustin de la vie de laquelle vous me demandez mon sentiment, écrit Marie de l'Incarnation au Père Poncet en 1670, je vous dirai entre vous et moi que je ne suis pas trop savante en ses affaires. Je sais seulement qu'à son extérieur, elle était dans la vie commune comme une bonne religieuse doit être. Lorsqu'elle était en santé (car elle était presque toujours malade) elle était une fidèle observatrice de ses règles. Mais depuis que j'ai su les étranges tentations et les persécutions atroces que les démons lui avaient suscitées jour et nuit l'espace de seize ans, j'ai cru que c'était là sa plus grande maladie... J'ai entendu de Monseigneur notre Prélat que cette bonne Mère était l'âme la plus sainte qu'il eût connue; il en pouvait parler comme savant, car c'est lui qui la dirigeait dans ces choses extraordinaires. Mais le Père Chastelain en sait plus qu'aucun autre, parce qu'il était son père spirituel et elle lui déclarait entièrement les secrets de son coeur. ${ }^{37}$

Il est possible que, chez Catherine, le psychisme ait été atteint, mais les maladies même de cet ordre n'ont jamais empêché une âme de parvenir à la sainteté et connaître une authentique vie mystique, plus difficile de ce fait à discerner, mais réelle.

La fondation de Montréal doit beaucoup aux mystiques; on peut la considérer comme l'oeuvre conjuguée de trois hommes qui n'ont jamais quitté le sol de France, deux laïcs: M. Le Royer de La Dauversière et le baron de Renty, un prêtre: $M$. Olier. L'idée première de la fondation de la colonie répond à un désir exprimé par les premiers missionnaires du Canada : attirer en Nouvelle-France des paysans, bons chrétiens, qui demeureraient auprès des Indiens, leur apprendraient à vivre de la

${ }^{37}$ Marie de l'Incarnation, Correspondance, p. 887. 
terre et sur la terre, les formant du même coup aux habitudes chrétiennes, afin de constituer outre-Atlantique une chrétienté sur le modèle de celle de France.

La brochure des "Véritables motifs des Messieurs et Dames de la Société de Notre-Dame de Montréal" a été composée en 1643, sous l'inspiration immédiate de La Dauversière ${ }^{38}$ qui avait rédigé en 1641 un premier schéma; elle a valeur de document autobiographique et il y a lieu d'en faire le plus grand cas, même et surtout si l'on considère les auteurs comme de pieux illuminés:

Le dessein de Montréal a pris son origine par un homme de vertu qu'il plût à la divine Bonté inspirer, il y a sept ou huit ans (donc en 1635), de travailler pour les Sauvages de la Nouvelle France dont il n'avait auparavant aucune particulière connaissance et, quelque répugnance qu'il en eût, comme chose par dessus ses forces, contraire à sa condition et nuisible à sa famille. Enfin, plusieurs fois poussé et éclairé par des vues intérieures qui lui représentoient réellement les lieux, les choses et les personnes dont il devait se servir... fortifié intérieurement à l'entreprendre comme service signalé que Dieu demandait de lui, il se rendit comme Samuel à l'appel de son Maître. ${ }^{39}$

La Dauversière est un élève et un disciple des Jésuites, mais il a été mis assez vite en relation avec $\mathbf{M}$. Olier et saint Vincent de Paul, et Renty devint son collaborateur et son ami; nous voyons donc confluer ici sur un fonds ignatien solide, le meilleur de l'École française et la spiritualité Carmélitaine conjuguée avec l'héritage bérullien, si tant est que Renty se laisse, lui aussi, enfermer dans une école de spiritualité.

La double conversion des Indiens à la vie chrétienne et à la vie sédentaire conçue sur le modèle français n'apparaissait pas comme une oeuvre aisée, mais les Associés étaient certains qu'il était possible d'aboutir à un résultat positif. L'Hôtel-Dieu de Montréal, confié à Jeanne Mance serait appelé à jouer un rôle important dans cette évolution vers une chrétienté indienne, encadrée par les Français.

\footnotetext{
${ }^{38}$ Voir G.-M. Oury, Jeanne Mance et le rêve de M. de la Dauversière, Chambrayles-Tours, 1983 (et bibliographie); id., «Le Fléchois Jérôme Le Royer de la Dauversière et l'utopie de Montréal ", dans La Province du Maine, t. 85, 1983, p. 150-62.

${ }^{39}$ Réédité en fac-similé par M.-Claire Daveluy, La Société de Notre-Dame de Montréal, Paris, 1965, p. 26.
} 
Sur place, en effet, les Associés de Montréal ont envoyé quelques grands chrétiens, Maisonneuve, le chef de la colonie, Jeanne Mance qui s'est offerte pour gérer le futur Hôtel-Dieu, puis un peu plus tard $\mathbf{M}$. d'Ailleboust, sa femme et sa belle-soeur.

Jeanne Mance s'est agrégée tardivement au groupe des hommes de Montréal; sa vocation canadienne lui est venue par d'autres voies: par un grand attrait intérieur et un appel, quand elle prit connaissance de la place faite aux femmes et aux religieuses dans la mission à Québec. À Langres déjà, elle se trouvait sous la direction spirituelle des Jésuites; à Paris, elle alla demander conseil au Père Charles Lalemant et surtout au Père Saint-Jure dont, jusqu'à sa mort, elle allait méditer l'oeuvre maitresse: De la connaissance et de l'amour de Notre Seigneur JésusChrist; une amitié spirituelle très profonde l'unit aussi au baron de Renty, relayée après la mort de celui-ci par une référence constante à sa Vie, écrite par le Père Saint-Jure. C'est à ces sources qu'elle dut de comprendre toujours davantage ce qu'impliquait sa propre voie d'abandon total, confiant, sans réserve aucune entre les mains de la Providence, dans la "complète désapropriation de soi" qu'elle pratiqua jusqu'à l'héroïsme.

Lorsque Marguerite Bourgeoys vint la rejoindre à Montréal avec la fameuse recrue de 1653 , la nouvelle venue avait déjà bénéficié en France d'influences spirituelles multiples: les deux plus fondamentales sont celles du saint Lorrain, Pierre Fourier, fondateur de la Congrégation de Notre-Dame qu'il avait dû orienter vers une forme semi-contemplative avec la clôture, et de Bérulle. La vision fondamentale qui semble avoir guidé Marguerite Bourgeoys à travers sa vie et sa mission, est le mystère de la Visitation de Marie, mystère de vie voyagère et conservante ${ }^{40}$.

Une présentation de son intuition est donnée par M. Montgolfier dans la Vie éditée en 1818 (mais écrite antérieurement) qui fait sans doute référence à des Écrits aujourd'hui perdus :

Notre Seigneur, en montant au ciel, a laissé sur la terre une espèce de congrégation de filles qui renferme tous les états et dont Marie était la première supérieure. Le Saint-Esprit dans l'Évangile nous a conservé les noms des principales Congréganistes qui, dans la suite, ont servi de modèles à toutes les communautés

\footnotetext{
${ }^{40}$ Voir L. Caza, La vie voyagère, conversante avec le prochain, Marguerite Bourgeoys, Montréal-Paris, 1982.
} 
de filles que Dieu a établies dans son Église. Ces Congréganistes étaient Madeleine et Marthe, disciples et amies du Sauveur du monde. La première devait être le modèle des Communautés religieuses qui, renfermées dans le cloître, s'appliquent principalement à la prière et à la contemplation; Marthe devait être le modèle de celles qui se consacrent à l'hospitalité; mais la Sainte Vierge qui était pour l'instruction, renfermait tout éminemment en sa propre personne. ${ }^{41}$

Saint François de Sales avait déjà été séduit par ie mystère de la Visitation et avait conçu l'Ordre religieux qu'il avait fondé, un peu sur le modèle que se proposait de réaliser Marguerite Bourgeoys; mais le vocabulaire et les modes d'expression sont bérulliens et c'est Bérulle qui reste la principale source d'inspiration.

Aussi Marguerite Bourgeoys se trouva-t-elle accordée avec le climat d'École française qui règna à Montréal à partir de l'arrivée des Sulpiciens; Olier avait été l'un des principaux animateurs de la Société des Messieurs et Dames de Montréal et les Sulpiciens de France la considéraient comme une sorte d'héritage familial.

Plus que personne avant lui, M. Olier avait contribué à rendre la doctrine de Bérulle accessible au grand nombre; il y avait d'autant plus de facilité qu'il l'avait connue lui-même principalement à travers Condren, c'est-à-dire déjà formulée dans une langue moins difficile, plus populaire, et allégée d'un certain nombre de développements théologiques. Ce bérullisme réduit, on pourrait presque dire appauvri, était susceptible d'exercer une influence beaucoup plus vaste et d'acquérir une audience considérable au sein du clergé et des fidèles. De tempérament très différent de Bérulle, Olier a trouvé des images très parlantes pour rendre vivantes auprès du grand nombre les splendeurs doctrinales de l'École française ${ }^{42}$.

Il serait d'un grand intérêt d'étudier pour elle-même la physionomie spirituelle de chacun des membres de la Compagnie de Saint-Sulpice qui vinrent au Canada dans la seconde moitié du XVII ${ }^{\mathrm{e}}$ siècle; mais ils ont surtout vécu l'héritage de M. Olier.

\footnotetext{
${ }^{41}$ Ibid., p. 54.

${ }^{42}$ Voir M. Dupuy, Se laisser à l'Esprit, Itinéraire spirituel de Jean-Jacques Olier, Paris, 1982; voir aussi J. Monier, Vie de Jean-Jacques Olier, Paris, 1914.
} 
On sait que le siège épiscopal de la Nouvelle-France a été, si l'on peut dire, disputé entre les Sulpiciens qui avaient mis en avant l'abbé de Queylus, et les Jésuites qui obtinrent la nomination de leur ancien élève, $\mathrm{M}^{\mathrm{gr}}$ François de Laval; celui-ci se disposait à partir en ExtrêmeOrient avec quelques compagnons pour répondre à un désir de la Congrégation de la Propagande; il changea donc de direction, mais non d'orientation, et resta très lié avec les fondateurs du Séminaire des Missions étrangères de Paris, voulant créer la réplique de celui-ci à Québec.

La dette de $\mathbf{M}^{\mathrm{gr}}$ de Laval à l'égard des Jésuites est considérable; il leur doit toute sa formation; à Paris, il a bénéficié de la direction du Père Bagot qui animait la Société des Bons Amis, formée de jeunes prêtres et clercs épris d'une vie sacerdotale pauvre et consacrée aux pauvres, évangélique au plein sens du terme. Mais il est aussi très attiré par le grand spirituel laïque, Jean de Bernières, auprès duquel il s'est retiré quelque temps avant sa nomination épiscopale. Bernières était résolument mystique par son orientation et se fit si bien l'apôtre d'une certaine passivité, que son Chrétien intérieur a fait l'objet d'une condamnation lors du conflit Bossuet-Fénelon, avec tous les ouvrages qui prônaient celle-ci ${ }^{43}$. Il est probable qu'il avait lui-même manqué de discrétion; saint Jean Eudes déclare que Marie de Vallée lui avait dit à plusieurs reprises que «autant d'âmes que (Bernières) mettait dans la voie de l'oraison passive - car c'est à Dieu à les y mettre - il les mettait dans le chemin de l'enfer" ${ }^{44}$. Il enseignait de préférence aux âmes, selon d'ailleurs une spiritualité très exigeante et de caractère un peu sombre, la consommation et l'unité en Dieu, l'anéantissement et la mort spirituelle, la vie cachée en Dieu pour une transformation en Jésus-Christ, la sainte folie de la croix, la pure passivité de l'âme pour une vie cachée en Dieu; à ses dirigés, il proposait la voie du pur amour, dégagé de toute considération personnelle, s'exprimant par une vie crucifiée, la pauvreté, le dépouillement, le renoncement absolu, les privations.

Cet idéal attirait certainement beaucoup d'âmes, puisque le Chrétien intérieur de l'aveu de Nicole, un anti-mystique déclaré, a eu plus de quarante éditions en seize ans.

\footnotetext{
${ }^{43}$ Voir. G. Souriau, La Compagnie du Saint-Sacrement à Caen, Deux mystiques normands au XVIr siècle, M. de Renty et J. de Bernières, Paris, 1913; R. Heurtevent, Les oeuvres spirituelles de Jean de Bernières, Paris, 1938.

${ }^{44}$ Saint Jean Eudes, Oeuvres, t. X, p. 439.
} 
$\mathbf{M}^{\mathrm{gr}}$ de Laval n'était pas lui-même un mystique, mais le fait de son amitié persévérante pour le groupe des disciples de Bernières, montre à l'évidence que son ascétisme n'était pas fermé et qu'il était parfaitement apte à en comprendre les dépassements.

L'empreinte personnelle qu'il a laissée à Québec est à l'image de sa vie : ardeur missionnaire, désintéressement, amour de la pauvreté, sens de l'accueil, importance de la vie intérieure, humilité et pénitence. Il a tenté de faire passer tout cela dans une Institution, le Séminaire des Missions étrangères de Québec qu'il concevait comme une véritable maison commune du clergé séculier missionnaire, celui-ci étant invité à mettre en commun toutes ses ressources dans un esprit de fraternité imité de la première communauté de Jérusalem au lendemain de la Pentecôte ${ }^{45}$.

Pour connaître dans toute son étendue ce que fut le sentiment religieux en Nouvelle-France au XVII siècle, il faudrait tenter de voir non pas seulement comment les valeurs chrétiennes, véhiculées par les différents courants religieux et spirituels, ont été vécues par les prêtres, religieux et séculiers, et les âmes d'élite, religieuses ou laïques, non pas seulement comment elles ont été enseignées et monnayées par la médiation de dévotions populaires (pratiquées d'abord très sincèrement par ceux qui les inculquaient): dévotion à saint Joseph, à la Sainte Famille, à l'Enfant Jésus, Roi des coeurs, à sainte Anne...; mais comment elles ont été reçues au fait par le peuple fidèle, tant du côté des Indiens convertis (et ici Kateri présente un sommet), que du côté des Français établis au Canada. Mais cela suppose une longue enquête, difficile à mener, comme tout ce qui touche la vie spirituelle des simples, connue

\footnotetext{
${ }^{45}$ Voir N. Baillargeon, Le Séminaire de Québec sous l'épiscopat de $M^{\text {pr }}$ de Laval, Québec, 1972, p. 163.
} 
seulement par ses manifestations extérieures, souvent très "formelles", et la matière abordée dans la présente étude était déjà surabondante. L'une des meilleures enquêtes dans cette direction est celle menée par Jean Simard et publiée en 1976: Les dévotions de l'École française et les sources de l'imaginerie religieuse en France et au Québec ${ }^{46}$. Elle ne prétend pas couvrir toute la vie religieuse populaire, et le champ reste ouvert aux recherches.

\author{
Dom Guy-Marie OURY \\ moine de Solesmes \\ Benedictine Monastery \\ Westfield, Vermont, U.S.A.
}

${ }^{46} \mathrm{~J}$. Simard, Une iconographie du clergé français au XVIF siècle, Les dévotions de l'École française et les sources de l'imagerie religieuse en France et au Québec au XVIF siècle, Québec, 1976; dans une direction analogue: P. Hurtubise, "Aspects doctrinaux de la dévotion à la Sainte Famille en Nouvelle-France», dans Eglise et Théologie, t. 3, 1972, p. 45-68; pour saint Joseph, on pourra consulter plusieurs études dans les Cahiers de Joséphologie. 